\title{
INFLUÊNCIA DA OSCILAÇÃO DECADAL DO PACÍFICO E DO PADRÃO PACIFIC SOUTH AMERICA NO CLIMA DA AMÉRICA DO SUL PARA O PERÍODO 1970-2003
}

\author{
SILVA, Maria Elisa Siqueira - mariaelisa.siqueirasilva@gmail.com \\ Professora do Dep. de Geografia da Faculdade de Filosofia, Letras e Ciências Humanas \\ (FFLCH) da Universidade de São Paulo. Programa de Pós-Graduação em Geografia Física USP \\ SILVA, Carlos Batista - krlosbatist@gmail.com \\ Bolsista CAPES do programa de Pós-Graduação em Geografia Física da Faculdade de \\ Filosofia, Letras e Ciências Humanas (FFLCH) da Universidade de São Paulo. Programa de \\ Pós-Graduação em Geografia Física USP
}

\begin{abstract}
RESUMO Este estudo tem como objetivo identificar os padrões climáticos de circulação e precipitação sobre a América do Sul durante as fases negativa e positiva da Oscilação Decadal do Pacífico - ODP, entre 1970 e 2003, associado-os aos modos de oscilação de baixa frequência no Pacífico Sul, para dados mensais da renalise I do NCEP-NCAR, reanálise do JRA-55 e dados de precipitação do GPCC. Os resultados mostram que os dados do NCEPNCAR e JRA-55 são bastante similares indicando anomalia positiva e negativa de altura geopotencial em altos níveis troposféricos sobre o sudeste da América do Sul, nas fases negativa e positiva da ODP, favorecendo, respectivamente, condições mais secas e mais úmidas que o normal para esta região. Associados a estes padrões de geopotencial, anomalias anticilônicas e ciclônicas de circulação em altos e baixos níveis são observadas nas fases negativa e positiva da ODP. A variabilidade da precipitação nas fases negativa e positiva da ODP é mais significativa no setor leste da América do Sul, sendo observada respectivamente menos e mais precipitação na faixa noroeste-sudeste do setor central do continente sulamericano.
\end{abstract}

PALAVRAS-CHAVE: fases da Oscilação Decadal do Pacífico; circulação sobre a América do Sul; modos de baixa frequência no Pacífico Sul

INFLUENCE OF PACIFIC SWING DECENNARY AND STANDARD OF PACIFIC SOUTH AMERICA MEASURE IN SOUTH AMERICA CLIMATE FOR THE PERIOD 1970-2003

ABSTRACT The aim of this study is identify the climatic patterns for circulation and precipitation at South America for negative and positive Pacific Decadal Oscillation, PDO, phases, between 1970 and 2003, associating them to the Pacific-South America, PSA, modes over South Pacific, for monthly data of NCEP-NCAR and JRA-55 reanalysis and GPCC precipitation data. Results show many similarities between NCEP-NCAR and JRA-55 datasets, and indicate positive and negative geopotencial heigh anomalies over the southeastern South America region, in high and low tropospheric levels, resectively for negative and positive PDO phases, favouring drier and wetter climatic condition in this region. Associated with the geopotencial high anomalous patterns, anticyclonic and cyclonic anomalies, on high and low levels are observed during negative and positive PDO phases. The precipitation variability during negative and positive PDO phases is more significant over the central-eastern South America, where it is observed less and more precipitation in the northwest-southeast belt placed in the central area of southamerican continent respectively on negative and positive PDO phases.

KEY WORDS: Pacific Decadal Oscillation phases, South America circulation, South Pacific low frequency modes

\section{INTRODUÇÃO}

Variações oceânicas no Oceano Pacífico têm grande potencial de induzir o clima global (Walker e Bliss, 1932; Bjerknes, 1969; Hoskins e Karoly, 1981; Horel e Wallace, 1981, Karoly, 1989; Mo e Nogués-Paegle 2001). Muitos estudos indicam a perturbação climática na América do Sul causada pela propogação de ondas de baixa frequência no Pacífico Sul (Mo e Ghil, 1987). O padrão mais expressivo de circulação de baixa frequência nas médias e altas latitudes do hemisfério sul foi observado por Mo e White (1985). Este padrão é também bem descrito por Mo e 
Ghil (1987) e por Mo e Nogués-Paegle (2001), sendo associado aos modos quasiestacionários do hemisfério sul, e, caracterizado por trens de onda do Pacífico equatorial central em direção ao continente sulamericano, conhecido como padrão Pacific South America, PSA. Anteriormente, vários outros estudos foram desenvolvidos sobre os padrões de circulação de baixa frequência para o hemisfério norte (Wallace e Gutzler, 1981; Horel e Wallace, 1981; Hoskins e Karoly, 1981). Estes estudos permitiram o melhor entendimento dos padrões quasi-estacionários entre médias e altas latitudes do hemisfério norte, e, padrões de teleconexões associados ao papel das fontes de calor nos trópicos (Wallace e Gutzler, 1981). O papel das fontes de calor sobre os padrões de circulação de baixa frequência na América do Sul foi apresentado por Karoly (1989), representando uma grande contribuição aos resultados de Mo e Ghil (1987).

Karoly (1989) mostrou a presença de trens de onda de baixa frequencia através do Pacífico Sul com dados de altura geopotencial para três eventos de El Niño. Baseando-se em um modelo barotropico. Hoskins e Ambrizzi (1993) mostraram o desenvolvimento de guias de trens de onda devido a fontes de calor em torno do globo, e, particularmente, os desenvolvidos sobre o Pacífico Sul devido às fontes de aquecimento no Pacífico equatorial. Kidson (1999) e Mo (2000) descreveram o padrão característico da propagação de trens de onda de baixa frequência sobre 0 Pacífico Sul, o padrão Pacific South America, PSA. Mo e Higgins (1998) e Mo e Nogués-Paegle (2001) identificam a importância dos padrões Pacific South America 1, PSA 1, e Pacific South America 2, PSA 2, representados pelos primeiros modos de Funções Ortogonais Empiricas (EOF, em inglês) aplicadas a dados de vento, como padrões de trens de onda relacionados a escala interanual e quase-bianual, respectivamente.

Mo e Nogués-Peagle (2001) relacionaram o modo PSA1 com a temperatura da superfície do mar (TSM) sobre áreas do Pacífico equatorial mais a oeste, associando-o à variabilidade de ENOS (El Niño-Oscilação Sul). Mo e Nogués-Peagle (2001) sugeriram que os modos PSA1e PSA2 sobre a América do Sul estão relacionados ao estado básico da atmosfera, embora eles também os associem à teoria de propagação de ondas devido à presença da fonte de calor equatorial, assim como discutem Bjerknes (1969), Wallace e Gutzler (1981), Karoly (1989) e Grimm et al., (2009). A variabilidade da precipitação na América do Sul também é associada aos modos PSA1 e PSA2 por Mo e Nogués-Peagle (2001). Silva et al. (2016) identificam padrões similares aos do PSA, referidos por Mo e Higgins (1998), para o hemisfério sul, para as distintas fases da Oscilação Decadal do Pacífico (ODP), entre 1970 e 2003, através de dados filtrados de geopotencial e vento. Estes resultados indicam a associação entre oscilações de baixa frequência sobre o Pacífico (e.g., ODP) e padrões característicos de circulação sobre a América do Sul.

Focados na escala decenal, outros estudos têm indicado a associação entre o clima da América do Sul e a Oscilação Decadal do Pacífico, particularmente considerando a variabilidade da precipitação. A ODP é caracterizada pela oscilação da TSM, em escala decadal (Latif e Barnett, 1994), entre áreas tropicais/equatoriais e extratropicais da bacia do Pacífico Norte, influenciando padrões climáticos ao redor do globo (Mantua et al., 1997; Mantua e Hare, 2002). Robertson e Mechoso (2000) associaram o aumento de precipitação e vazão de rios sobre o sudeste da América do Sul e sudeste da Amazônia com a fase positiva da ODP, de 1978 a 1996. Marengo (2004) mostrou a diminuição da precipitação no norte da Amazônia depois de 1976-1977, também a associando com a fase positiva da PDO. Kayano e Andreoli (2004) mostraram a associação da chuva sobre o nordeste do Brasil com a variabilidade da ODP, através da aplicação da técnica de ondeletas, mostrando correlações significativas para a escala decadal ( $r>0,8)$. Silva (2012) e Silva et al. (2016) associaram o aumento (diminuição) da vazão de rio na região do Pantanal com a fase positiva (negativa) da ODP no período de 1970 a 2003. Neste último 
estudo, os autores também mostraram padrões médios distintos da circulação sobre o Pacífico Sul para fases opostas da ODP. Associado aos distintos padrões de circulação sobre o Pacífico Sul, Silva et al. (2016) encontraram condições climáticas significativamente distintas para a América do Sul. Durante as fases negativa (positiva) da ODP, identificaram, em altos níveis, anomalias positivas (negativas) da altura geopotencial sobre o sudeste do Brasil e Oceano Atlântico Sul adjacente. Também identificaram padrões particulares de anomalia de vento, em baixos níveis, sobre o centro-leste da América do Sul, com anomalia anticiclônica durante as fases negativas e ciclônica durante a fase positiva da ODP. Para explicar as anomalias de vazão na região do Pantanal, durante o período de 1970 a 2003, Silva et al. (2016) basearam-se em condições atmosféricas dos dados da reanálise I do NCEP-NCAR (Kalnay et al., 1996).

Os resultados de Mo e Nogués-Peagle (2001) mostram os padrões característicos de circulação atmosférica sobre o Pacífico Sul e América do Sul para o período de verão de 1949 a 2000, sem levar em consideração períodos específicos da ODP. Neste artigo, dá-se atenção à definição de padrões médios de circulação atmosférica sobre o Pacífico e América do Sul para as fases distintas da ODP entre 1970 e 2003, considerando uma fonte adicional de dados, a reanálise JRA-55 (Kobayashi et al., 2015).

\section{DADOS E MÉTODOS}

Dados mensais de vento, altura geopotencial e precipitação, de janeiro de 1970 a dezembro de 2013, foram considerados no presente estudo. Os dados atmosféricos foram obtidos da reanálise I do NCEP-NCAR (Kalnay et al., 1996) e da reanálise JRA-55 (Kobayashi et al., 2015). Os dados de precipitação foram obtidos do Global Precipitation Climatology Center - GPCC (Schneider et al., 2011). Os dados do JRA55 foram considerados de forma a permitir a comparação com os resultados de Silva et al. (2016), que usaram dados da reanálise I do NCEP-NCAR. O índice mensal da PDO foi obtido da NOAA (http://www.esrl.noaa.gov/psd/data/climateindices/list/) e é definido pelo modo de EOF aplicado a TSM mensal para o Oceano Pacífico Norte, a norte de $20^{\circ} \mathrm{N}$ (Mantua et al., 1997; Mantua Hare, 2002; Mochizuk et al., 2010; Krishnamurthy e Krishnamurthy, 2014). Os conjuntos de dados apresentam as seguintes resoluções espaciais: NCEP-NCAR, 2,5 graus; JRA, 1,25 graus; e a precipitação do GPCC apresenta resolução de 1,0 grau (Adler et al., 2003).

Para reter a oscilação de baixa frequência, e, então, a propagação de ondas de Rossby, os dados de ventos e altura geopotencial, para os dois conjuntos, foram suavizados com o filtro passa-baixa de Lanczos (Duchon, 1979). Seguindo os resultados de Silva et al. (2016), as análises apresentadas aqui referem-se às fases da ODP entre 1970 e 2003, definidas pelos subperíodos 1970-1976 (fase negativa), 1977-1996 (fase positiva) e 1997-2003 (fase negativa). Os padrões climáticos associados com as fases da ODP são analisados com base em mapas médios e a significância estatística foi calculdada considerando-se o teste t-Student. Além do padrão climático na América do Sul, a propagação de ondas de baixa frequência do Pacífico equatorial para a América do Sul é identificada em cada fase da ODP.

\section{RESULTADOS}

Os dois conjuntos de dados de reanálises, NCEP-NCAR e JRA-55, indicam padrões semelhantes de baixa frequência entre o Pacífico equatorial e a região subtropical da América do Sul, para as três fases da ODP, 1970-1976, 1977-1996 e 19972003, como mostrado na Fig. 1. Nas fases negativa e positiva da ODP, os dois 
conjuntos de dados apresentam comportamentos opostos. Durante as fases negativas, $1970-1976$ e 1997-2003 ( $1^{\circ}$ e $3^{\circ}$ subperíodos), observa-se anomalia negativa de altura geopotencial em $250 \mathrm{hPa}$ sobre o Pacífico central equatorial associada com o resfriamento médio da camada atmosferérica subjacente neste período, tanto no hemisfério sul quanto no norte, e, anomalias positivas sobre o centro-leste da América do Sul, incluindo seu setor sudeste. Durante a fase positiva ( $2^{\circ}$ subperíodo), 1977-1996, observa-se o padrão contrário, caracterizado por anomalias positivas de altura geopotencial em $250 \mathrm{hPa}$ sobre o Pacífico central equatorial e anomalias negativas sobre o sudeste da América do Sul. Tanto na fase negativa quanto na positiva, os padrões de trens de onda de baixa frequência, associados à propagação de ondas de Rossby, no Pacífico Sul, são caracterizados de forma similar ao indicado por Mo e Ghil (1986), Karoly (1989), Mo e NoguésPaegle (2001), Ding et al. (2011), Irving e Simmonds (2016).

Em geral, a anomalia média de altura geopotencial em $250 \mathrm{hPa}$, no segundo subperíodo, apresenta valores menores do que os observados para o primeiro e terceiro subperíodos, para os dois conjuntos de dados considerados, o que deve ser resultado do maior comprimento da série temporal para este período intermediário. O segundo período é constituído por 20 anos enquanto o primeiro e terceiro subperíodos são constituídos por seis e sete anos, respectivamente. Durante o terceiro subperíodo, a anomalia de altura geopotencial em $250 \mathrm{hPa}$ apresenta os padrões mais distintos dentre as fontes consideradas. Enquanto o padrão médio de trem de onda do Pacífico equatorial para o Pacífico Sul é bastante similar para as duas fontes de dados (Fig. 1C,C*), a evolução para a América do Sul mostra diferenças mais significativas. Os dados da reanálise I do NCEP-NCAR mostram anomalia média negativa de altura geopotencial no sul da América do Sul, no 30 subperiodo, enquanto que os dados da reanálise JRA-55 mostram sinal positivo nas mesmas áreas. Ainda assim, o sinal de anomalia de altura geopotencial observado no sudeste da América do Sul é positivo para as duas fontes de dados, contribuindo ambos como forçantes semelhantes. Apesar do sudeste da América do Sul ser uma região distante da forçante do Pacífico equatorial, mostra, durante fases distintas da ODP, padrões atmosféricos com sinais invertivos. Em outras palavras, pode-se dizer que a fonte de calor do Pacífico equatorial seja suficientemente forte para perturbar fisicamente o clima médio do sudeste da América do Sul. Segundo Bromwich et al. (2007), a comparação entre as reanálises do NCEP-NCAR e JRA-55 apresenta diferenças mais significativas nas regiões polares. Certamente, análises adicionais devem contribuir para aprofundar o entendimento sobre as diferenças observadas dentre as fontes de dados consideradas.

Por outro lado, o principal objetivo deste estudo é apontar as semelhanças entre as fontes de dados como um forma de corroborar os padrões observados em Silva et al. (2016), uma vez que seus resultados indicam a existência de padrões climáticos mais secos ou chuvosos no sudeste da América do Sul aparentemente bem associados à variabilidade da Oscilação Decadal do Pacífico (Mantua et al., 1997). Durante a fase negativa da ODP, $1^{\circ}$ e 30 subperíodos (1970-1976 e 1997-2003), a região centro-leste da América do Sul, que inclui o sudeste do Brasil, apresenta anomalia positiva de altura geopotencial em níveis altos (figs. 1a,c e 1a*,c*) para as fontes de dados do NCEP-NCAR e JRA-55. Por outro lado, a fase positiva da ODP, observada durante o $2^{\circ}$ subperíodo (1977-1996), apresenta anomalia negativa de altura geopotencial em altos níveis sobre a mesma área, sudeste do Brasil, para os dois conjuntos de dados considerados, como mostrado na Fig. 1b, b*. Padrões caracterizados por anomalias positiva (negativa) de altura geopotencial em altos níveis sobre o sudeste do Brasil, durante a fase negativa (positiva) da ODP, podem estar associados à maior frequência de condições climáticas secas (úmidas), uma vez que a troposfera apresente tendência à barotropicidade. Coelho et al. (2015) elaborou uma consistente análise climática do período seco ocorrido em 2014, no sudeste do Brasil, quando houve déficit hidrico na região, em diversos municípios 
do Estado de São Paulo. Neste ano, a região sofreu com a falta de abastecimentos hídrico às residências urbanas, déficite na irrigação agrícola e na geração de energia elétrica, forçando o governo Federal a produzir mais energia elétrica a partir de termoelétricas. Neste estudo, os autores mostraram o desenvolvimento de uma anomalia anticiclônica por toda a troposfera, sobre o sudeste brasileiro, o que levou a um período anomalamente seco. Assim, considera-se que a análise do perfil vertical da troposfera sobre o sudeste do Brasil durante os três subperíodos considerados entre 1970 e 2003 deve contribuir para caracterizar o aspecto barotrópico nas fases da ODP.
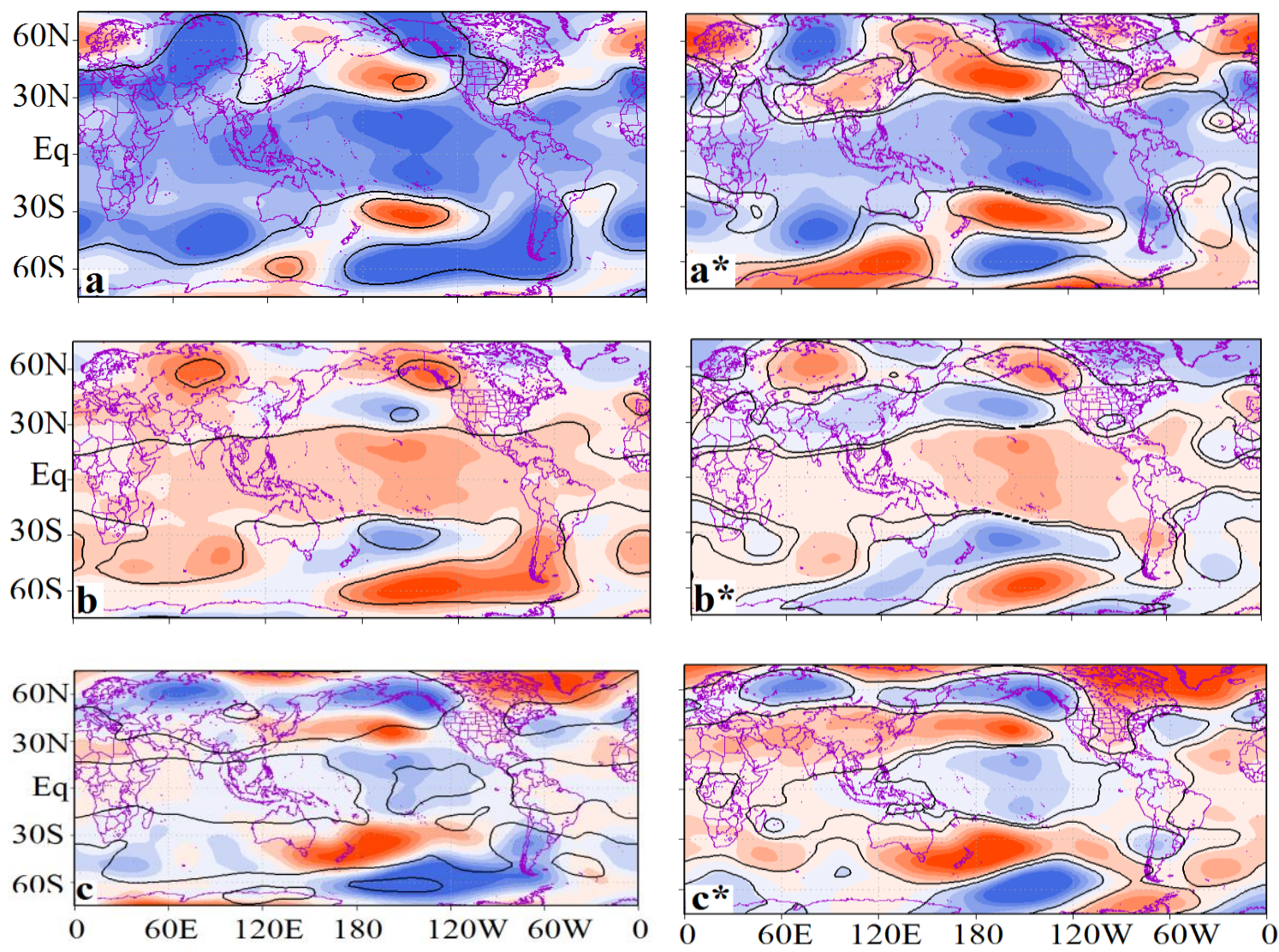

Figura 1 Anomalia de altura geopotencial (mgp) para os três subperíodos (a) 1970-1976, (b) 1977-1996 e (c) 1997-2003 para dados da reanálise I do NCEP-NCAR. (a*), (b*) e (c*) referem-se aos mesmos subperíodos, mas para a reanálise do JRA-55. Isolinhas pretas indicam significância estatística maior que 95\%.

O perfil vertical da correlação linear entre o índice de PDO e a altura geopotencial, para a faixa latitudinal $25^{\circ} \mathrm{S}-23^{\circ} \mathrm{S}$, mostrado na Fig. 2, indica a característica barotrópica da troposfera para a região sudeste do Brasil durante as fases da ODP. Esta faixa latitudinal, definida entre $25^{\circ} \mathrm{S}$ e $23^{\circ} \mathrm{S}$, foi escolhida por duas razões específicas: primeiramente, do ponto de vista climático, abrange um dos centros de ação do PSA sobre o sudeste da América do Sul (a despeito de apresentar fraca intensidade em comparação aos demais centros), e, do ponto de vista geográfico, por coincidir com a região mais densamente ocupada e por apresentar a maior concentração econômica do país. Durante a fase positiva (negativa) da ODP, anomalias negativas de altura geopotencial são persistentes da superfície até 250 $\mathrm{hPa}$, sobre o sudeste da América do Sul, entre $50^{\circ} \mathrm{W}$ e o meridiano de Greenwich, o que é visto pelos valores de correlação linear da Fig. 2. A Figura 3 mostra o perfil 
vertical médio da anomalia da componente meridional do vento em cada subperíodo, para as duas fontes de dados, entre $180^{\circ} \mathrm{W}$ e o meridiano de Greenwich. Em geral, os quadros da Fig. 3 mostram padrões barotrópicos para as duas fontes de dados, durante os três subperíodos. Durante as fases negativas, os dados mostram anomalias para sul, a oeste de $45^{\circ} \mathrm{W}$, e anomalias para norte, a leste desta longitude, por toda a troposfera, o que está associado a uma anomalia anticiclônica centralizada em $\left(50^{\circ} \mathrm{W} ; 18^{\circ} \mathrm{S}\right)$ (indicado na Fig. 4a). Os dados de vento do JRA-55 mostram anomalias menos intensas do que as do NCEP-NCAR (Fig. 3a), principalmente nos níveis baixos, para o $1^{\circ}$ subperíodo. Durante a fase positiva da PDO, a anomalia da componente meridional do vento na região sudeste do Brasil apresenta um sinal oposto ao observado nas fases negativas, tal como esperado pelos resultados mostrados nas Figuras 1 e 2: a oeste de $47^{\circ} \mathrm{W}$, a anomalia predominante é para norte e, a leste desta longitude, a anomalia predominante é para sul, por todos os níveis troposféricos.

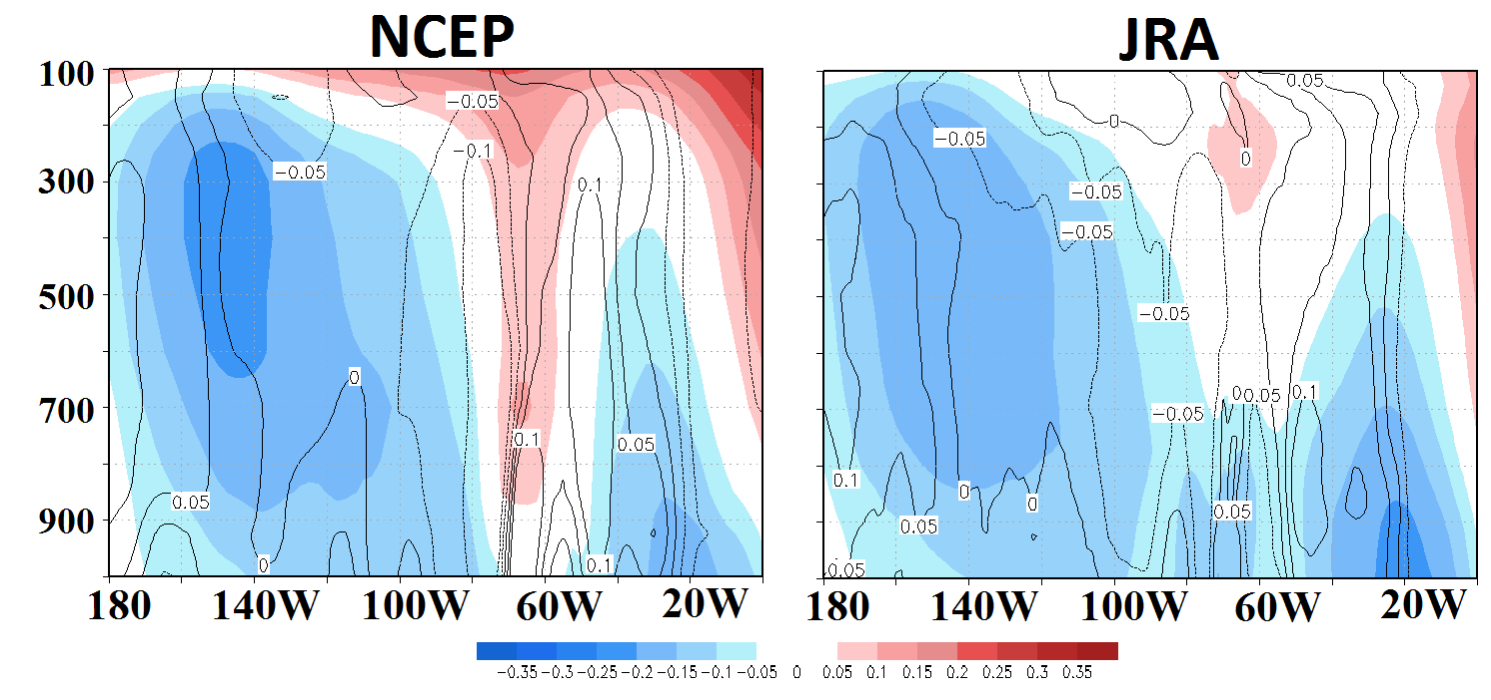

Figura 2 Perfil vertical (1000-100 hPa) de correlação linear entre o índice de ODP e altura geopotencial (em cor), entre $25^{\circ} \mathrm{S}$ e $23^{\circ} \mathrm{S}$, para o perído $1970-2003$. Os valores sombreados são plotados apenas para significância estatística superior a 95\%. A componente meridional dc vento é indicada por isolinhas. 

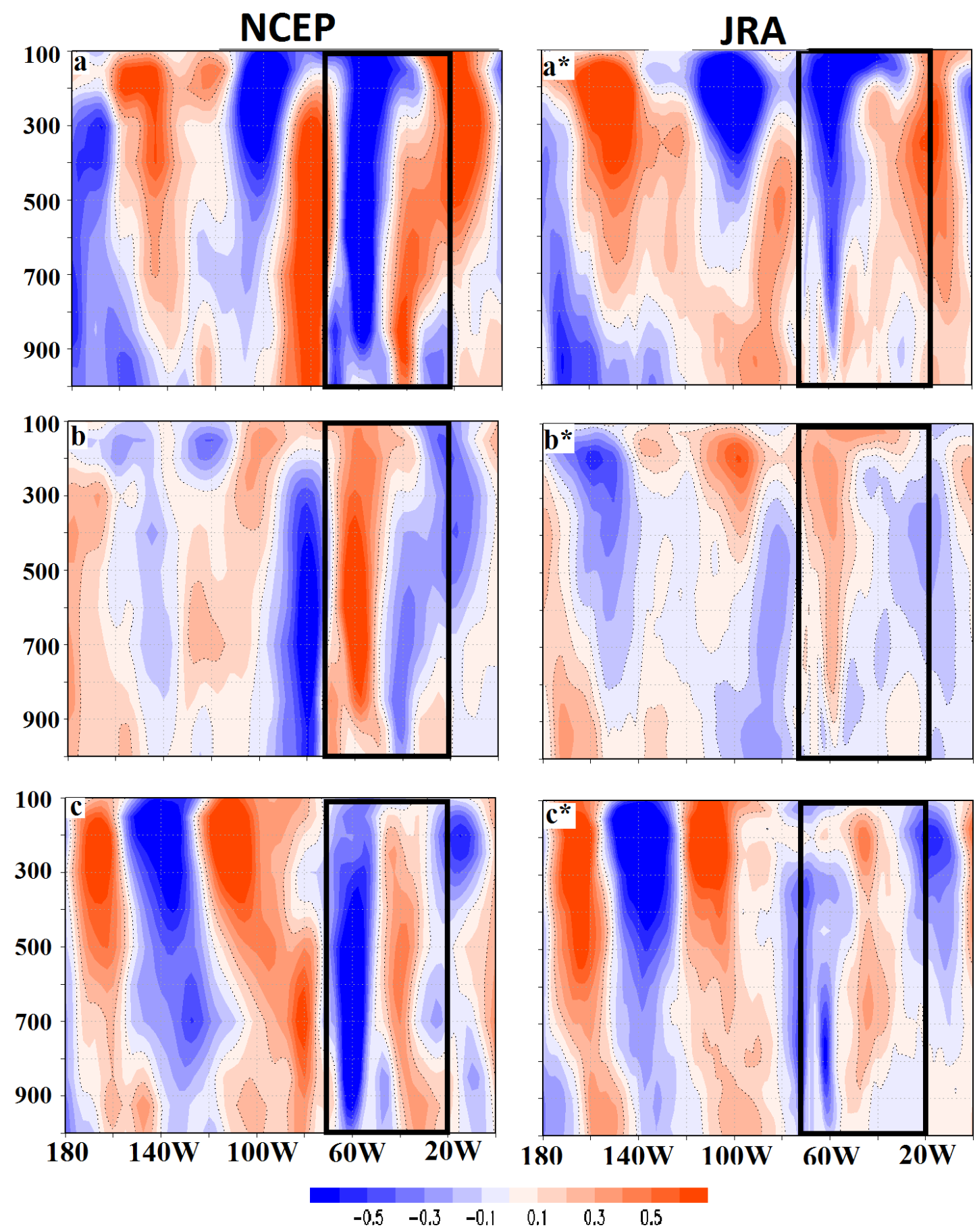

Figura 3 Perfil vertical da anomalia da componente meridional do vento ( $\mathrm{m} \mathrm{s}^{-1}$ ) para os subperíodos (a) 1970-1976, (b) 1977-1996 e (c) 1997-2003, para os dados do NCEP-NCAR. $\left(a^{*}\right),\left(b^{*}\right)$ e $\left(c^{*}\right)$ referem-se a dados do JRA-55.

Como mostrado por Silva et al. (2016) e outros autores, os padrões de circulação de baixa frequência associados com a Oscilação Decadal do Pacífico alcança o sudeste do Brasil e influencia o clima da América do Sul. Para compreender os padrões de circulação atmosférica característicos e favoráveis ao estabelecimento de condições climáticas distintas em fases distintas da ODP, no período 1970-2003, 
analisa-se, na Fig. 4, a anomalia do vetor vento sobre a América do Sul na baixa (850-600 hPa) e alta (500-200 hPa) troposfera, em relação ao valor médio para todo o período, para as fontes de dados consideradas.

Em geral, tanto os dados da reanálise I do NCEP-NCAR como os dados do JRA-55 mostram anomalias anticiclônicas (Fig. 4a,c e 4a*,c*; 5a,c e 5a*,c*) e ciclônicas (Fig. 4b e 4b*; $5 b$ e 5b*) sobre o centro-leste da América do Sul, respectivamente durante as fases negativa e positiva da ODP, tanto em baixos quanto em altos níveis. Este resultado concorda com o obtido por Silva et al. (2016). Em baixos níveis (850-600 hPa), o padrão de anomalias ciclônica e anticiclônica são mais restritos espacialmente; por outro lado, em níveis altos, os padrões de anomalia da circulação cobrem áreas maiores, alcançando áreas adjacentes do Atlântico Sul sudoeste. O padrão de anomalia ciclônica observado no $2^{\circ}$ subperíodo apresenta valores mais baixos do que os relativos ao $1^{0}$ e $3^{\circ}$ subperídos, o que deve ser explicado pelo tamanho das séries temporais para os diferentes subperíodos, como mencionado anteriormente. 

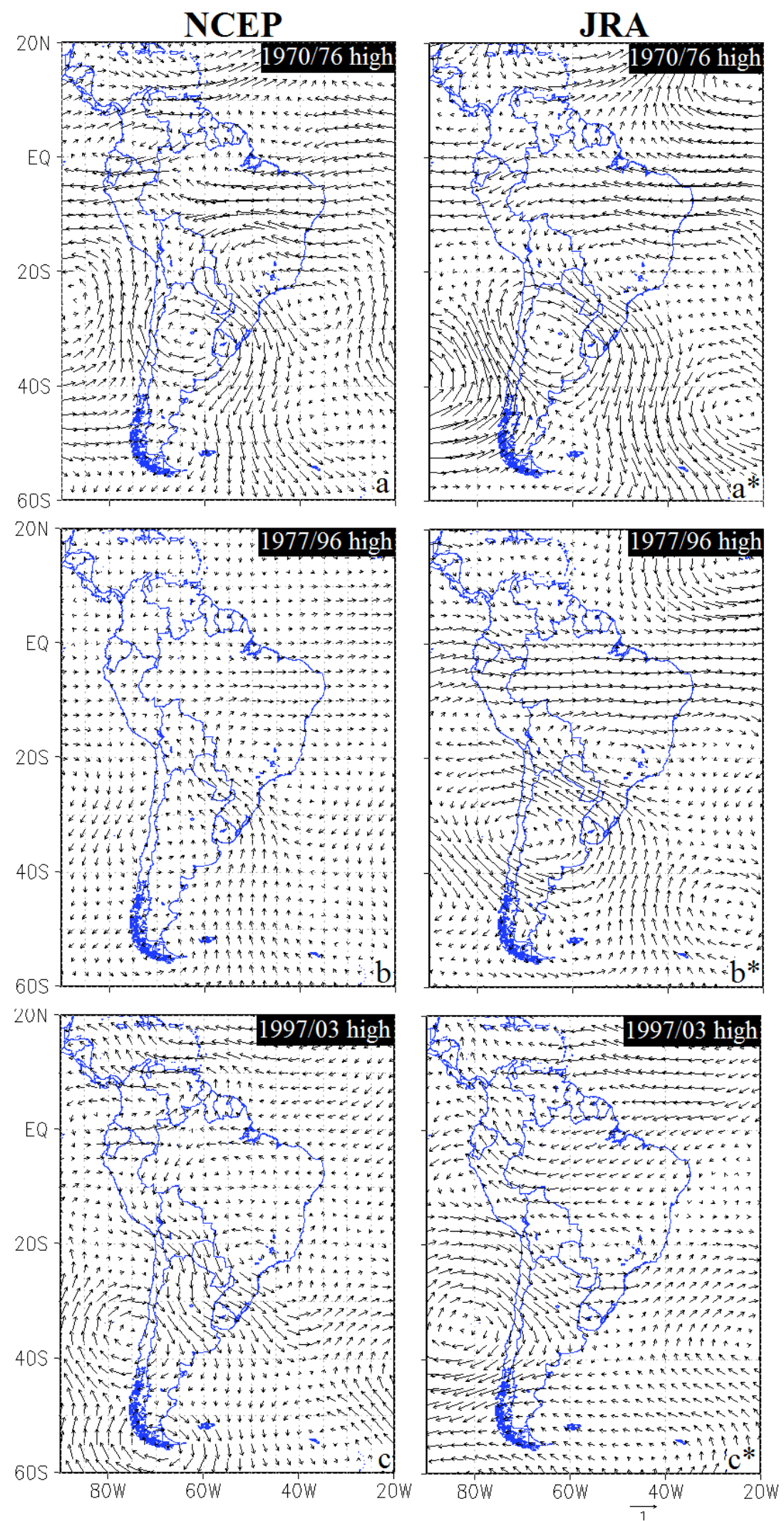

Figura 4 Anomalia média do vetor vento para os subperíodos $1^{\circ}(1970-1976), 2^{\circ}$ (19771996) e $3^{\circ}$ (1997-2003), em altos (500-200 hPa) níveis, para as reanálises do NCEP-NCAR e JRA-55. 



Figura 5 Idem à Fig. 4, mas para baixos $(850-600 \mathrm{hPa})$ níveis.

Com a análise dos dados de ventos e altura geopotencial das reanálises do NCEPNCAR e JRA-55 conclui-se que as fases negativas da ODP, do período de 1970 a 2003, estiveram caracterizadas pela intensificação do escoamento de leste em altos 
e baixos níveis no nordeste da América do Sul e oceano Atlântico tropical adjacente, e, também pela intensificação do escoamento de norte sobre o centro do continente, o que é particularmente importante em baixos níveis uma vez que o escoamento de norte leva para sul ar carregado de umidade. Ainda para as fases negativas, a intensificação dos ventos de leste no norte da América do Sul carrega mais umidade do Oceano Atlântico para áreas continentais. Por outro lado, a fase positiva da ODP é caracterizada pelo enfraquecimento dos ventos de leste (alíseos) sobre o nordeste da América do Sul e dos ventos de norte sobre a região central do continente. A análise de EOF aplicada a dados de vento do NCEP-NCAR mostra padrões opostos da anomalia da circulação do ar sobre o centro da América do Sul para as fases negativa e positiva da ODP, como mostrado na Fig. 6. A análise do coeficiente de expansão temporal do $4^{\circ}$ modo da análise de EOF mostra a subdivisão da série temporal em três períodos, que se aproximam dos subperíodos definidos pelas distintas fases da ODP neste estudo. Enquanto que Mo e NoguésPeagle (2001) mostram um padrão espacial similar de EOF aplicada a dados de vento para as estações de verão (DJF) do período de 1949 a 2000, sem associá-lo às fases da ODP, neste estudo é mostrada esta associação considerando todos os meses do ano (Fig. 6).

(a)

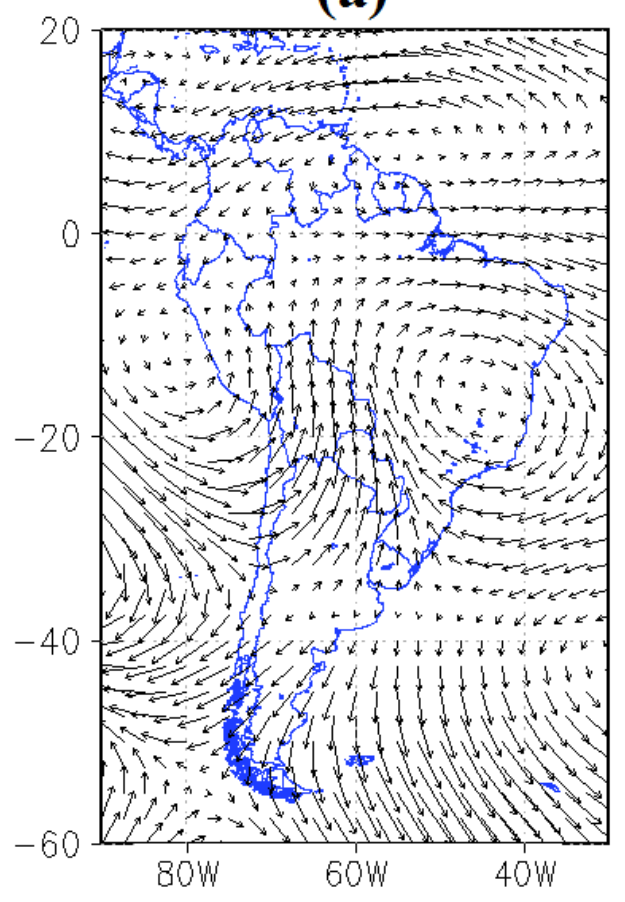

(b)

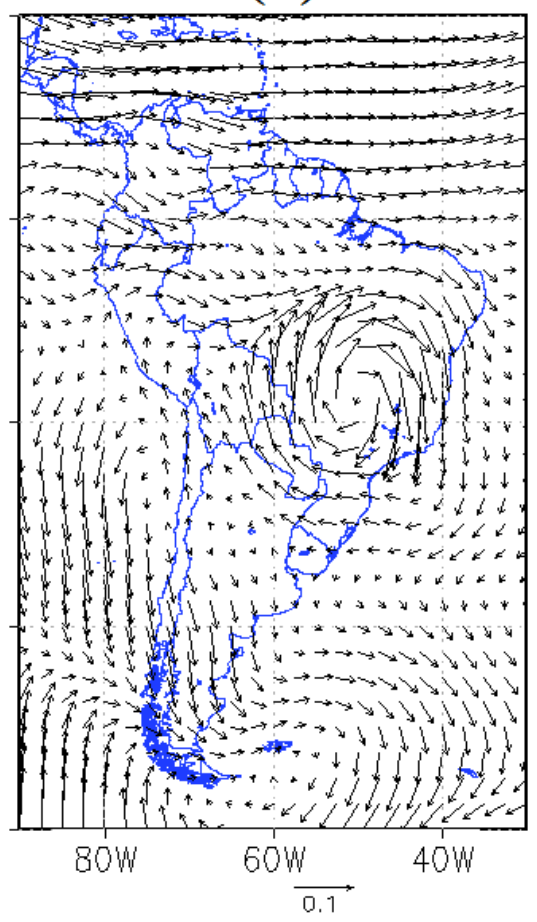

Figura $64^{\circ}$ modo de EOF aplicada a dados mensais de vento da reanálise I do NCEP-NCAR para os níveis atmosféricos (a) $850 \mathrm{hPa}$ e (b) $250 \mathrm{hPa}$, para o período 1970-2003.

Como indicado anteriormente, o padrão de vento observado nas três fases da ODP entre 1970 e 2003 deve estar associado ao padrão de anomalias de precipitação na América do Sul. A Fig. 7 mostra a anomalia média de precipitação do GPCC para cada subperíodo. A análise da precipitação para os três subperíodos é marcadamente distinta nas três fases da ODP. As fases negativas da ODP, 10 e 30 subperíodos, estão associadas a menos precipitação sobre o leste da faixa noroeste-sudeste alinhada sobre a região central da América do Sul, e, a mais precipitação sobre o sudeste da América do Sul, incluindo o sul e sudeste do Brasil e Uruguai. A fase positiva da ODP está associada com o padrão oposto nestas mesmas áreas, com mais precipitação sobre o setor leste da faixa noroeste-sudeste e menos precipitação no sudeste da América do Sul (Fig 7b). 
Uma questão que se pode fazer é: quais os processos da dinâmica atmosférica contribuem para prover os padrões de precipitação observado na América do Sul nas fases positiva e negativa da ODP? Considerando o padrão de anomalias de ventos (Figs. 4-6), pode-se supor que anomalias anticiclônicas sobre o centro-leste da América do Sul provêem um escoamento de norte mais intenso, com mais umidade sendo retirada do norte da América do Sul para áreas mais ao sul na fase negativa da ODP. Ao mesmo tempo, alíseos mais intensos devem contribuir para o transporte de umidade do Atlântico tropical para áreas continentais. $\mathrm{Na}$ fase positiva da ODP, anomalias ciclônicas são observadas no centro-leste da América do Sul enfraquecendo os alíseos nas áreas tropicais e o escoamento de norte sobre o centro do continente, o que deve provocar convergência de umidade na faixa noroeste-sudeste, provendo aumento da precipitação no centro da América do Sul. Respostas a estas indagações devem ser contempladas futuramente em um próximo estudo.

(a)

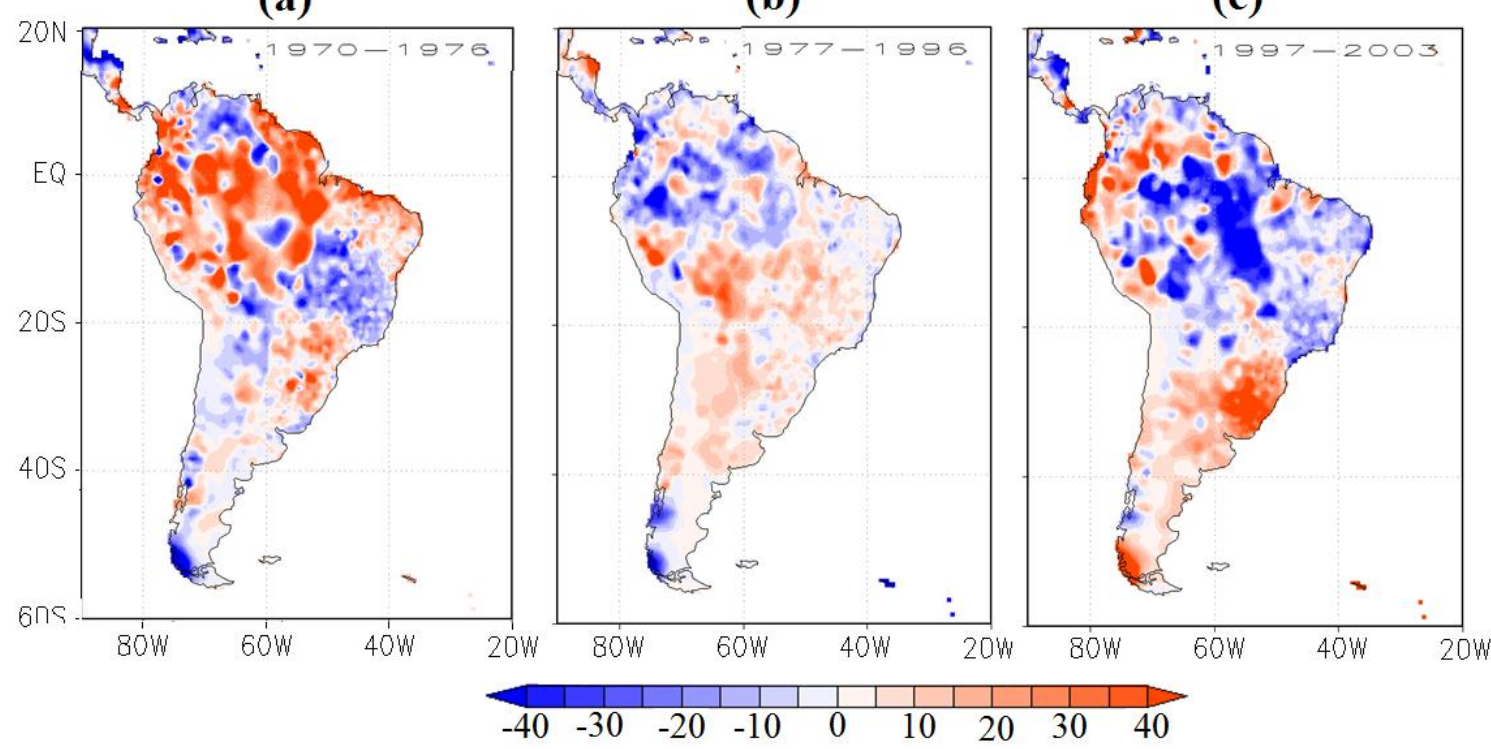

Figura 7 Anomalia média da precipitação mensal $(\mathrm{mm})$ do GPCC filtrada (Lanczos - passa baixo) para os subperíodos (a) $1^{\circ}$, (b) $2^{\circ}$ e (c) $3^{\circ}$, entre 1970 e 2003.

\section{CONCLUSÕES}

Neste estudo é realizada uma análise diagnóstica da anomalia média dos dados de altura geopotencial, vento e precipitação para os conjuntos de dados das reanálises do NCEP-NCAR (reanálise I), JRA-55 e dados do GPCC, associada com as fases da Oscilação Decadal do Pacífico entre 1970 e 2003, sobre a América do Sul. O objetivo deste estudo é estabelecer o padrão de circulação e precipitação nas distintas fases da ODP entre 1970 e 2003 a partir dos dados do NCEP-NCAR e JRA55 para, adicionalmente, comparar com os resultados obtidos por Silva et al. (2016). A análise dos dados do NCEP-NCAR e JRA-55 permite concluir que, em geral, eles produzem padrões similares de anomalias de vento e altura geopotencial para os subperíodos 1970-1976, 1977-1996 e 1997-2003. Anomalias de geopotencial em altos níveis mostram padrões opostos para fases opostas da ODP. Durante as fases negativas (positiva), o sudeste do Brasil é caracterizado por anomalias positivas de geopotencial que se extende por toda a troposfera. Os padrões de circulação observados sobre a América do Sul durantes as distintas fases da ODP são claramente associados ao padrão médio da propagação de ondas de baixa frequência no Pacífico Sul, contribuindo para a modulação climática na 
região. Os resultados obtidos neste estudo concordam com os modos PSA1 e PSA2, tal como indicado por Mo e Peagle (2001), obtidos pela análise de EOF, aplicada a dados diários de vento para os meses de verão de 1950 a 2000.

Os dados de anomalia de vento mostram anomalias anticiclônicas e ciclônicas sobre o centro-leste da América do Sul, respectivamente, durante as fases negativa e positiva da ODP. Anomalias anticiclônicas e ciclônicas observadas em altos níveis atmosféricos podem ser associadas a períodos secos e úmidos sobre a região sudeste do Brasil, assumindo uma atmosfera barotrópica. Os perfis verticais de anomalia de altura geopotencial e vento indicam um comportamento barotrópico sobre o sudeste da América do Sul, contribuindo para explicar os padrões úmidos e secos. Buscando por razões que levassem às condições climáticas secas observadas em 2014, no sudeste do Brasil, Coelho et al. (2015) mostraram a persistência de anomalias anticiclônicas e o comportamento médio barotrópico para a região. Considerando-se os resultados obtidos em Coelho et al. (2015) e os obtidos no presente estudo, sugere-se que o período seco de 2014 constitua parte de um período seco maior definido em escala decadal, atrelado à dinâmica atmosférica de baixa frequência.

Anomalias anticiclônicas e ciclônicas observadas, respectivamente, durante as fases negativa e positiva da ODP devem estar associadas aos primeiros modos de EOF do fluxo de umidade integrado verticalmente e mostrados por Mo e Nogués-Peagle (2001), e, como sugerido por estes autores, à variabilidade do ENOS. Apesar dos modos de EOF do fluxo de umidade integrado verticalmente, mostrados por Mo e Nogués-Peagle (2001), PSA1 e PSA2, indicarem padrões opostos sobre a América do Sul, contribuem com o mesmo sinal para a variabilidade da precipitação, o que é possível de ser identificado nos campos de divergência do ar em $200 \mathrm{hPa}$. Como o presente estudo considera o período 1970-2003, interno ao período considerado por Mo e Nogués-Peagle (2001), 1950-200, é razoável supor que os resultados obtidos aqui constituam um caso particular do primeiro. Assim, análises adicionais devem ser feitas para esclarecer a associação possível entre os resultados obtidos neste estudo e aqueles obtidos por Mo e Nogués-Peagle (2001).

As análises dos padrões de precipitação com os dados do GPCC mostram anomalias negativa e positiva sobre a faixa noroeste-sudeste do centro da América do Sul, respectivamente, sobre as fases negativas e positivas da ODP. A intensificação (enfraquecimento) do escoamento de norte sobre a região central da América do Sul está associada a anomalias negativas (positivas) de precipitação sobre a faixa noroeste-sudeste durante as fases negativa (positiva) da ODP. Por outro lado, as fases negativas da ODP são caracterizadas pela intensificação (enfraquecimento) do escoamento de leste dos alíseos sobre o nordeste da América do Sul e Atlântico tropical adjacente, levando mais (menos) ar úmido do Atlântico tropical para áreas continentais, em especial, sobre a faixa noroeste-sudeste do centro da América do Sul. A maior compreensão da eficiência dos processos de transporte de umidade entre o Atlântico tropical, a faixa noroeste-sudeste sobre o centro da América do Sul e a região centro-sul do continente durante fases distintas da ODP deve ser considerada em estudos futuros.

\section{Agradecimentos}

Os autores agradecem ao professor Tércio Ambrizzi (IAG/USP) e a pesquisadora Anita Drummont, da Universidade de Vigo, Espanha, pelas discussões e sugestões ao artigo. O segundo autor agradece o suporte financeiro efetuado pela CAPES. 


\section{REFERÊNCIAS}

Adler, R.F., G.J. Huffman, A. Chang, R. Ferraro, P. Xie, J. Janowiak, B. Rudolf, U. Schneider, S. Curtis, D. Bolvin, A. Gruber, J. Susskind, P. Arkin and E. Nelkin (2003): The Version-2 Global Precipitation Climatology Project (GPCP) Monthly Precipitation analysis (1979- present). J. Hydrometeorol., 4, 1147-1167.

Kayano, M. T. \& Andreoli, R. V. (2004). Decadal variability of northern northeast Brazil rainfall and its relation to tropical sea surface temperature and global sea level pressure anomalies. Journal of Geophysical Research: Oceans, 109(C11).

Bjerknes, J. A. B. (1969). Atmospheric teleconnections from the equatorial Pacific.

Bromwich, D. H., Fogt, R. L., Hodges, K. I., \& Walsh, J. E. (2007). A tropospheric assessment of the ERA-40, NCEP, and JRA-25 global reanalyses in the polar regions. Journal of Geophysical Research: Atmospheres, 112(D10).

Coelho, C. A., de Oliveira, C. P., Ambrizzi, T., Reboita, M. S., Carpenedo, C. B., Campos, J. L. P. S., ... \& Da Rocha, R. P. (2015). The 2014 southeast Brazil austral summer drought: regional scale mechanisms and teleconnections. Climate Dynamics, 1-16.

Ding, Q., Steig, E. J., Battisti, D. S., \& Küttel, M. (2011). Winter warming in West Antarctica caused by central tropical Pacific warming. Nature Geoscience, $4(6)$, 398-403.

Duchon, C. E. (1979). Lanczos filtering in one and two dimensions. Journal of Applied Meteorology, 18(8), 1016-1022.

Grimm, A. M., \& Ambrizzi, T. (2009). Teleconnections into South America from the tropics and extratropics on interannual and intraseasonal timescales. In Past Climate Variability in South America and Surrounding Regions (pp. 159191).Springer Netherlands.

Hoskins, B. J., \& Karoly, D. J. (1981). The steady linear response of a spherical atmosphere to thermal and orographic forcing. Journal of the Atmospheric Sciences, 38(6), 1179-1196.

Hoskins, B. J., \& Ambrizzi, T. (1993). Rossby wave propagation on a realistic longitudinally varying flow. Journal of the Atmospheric Sciences, 50(12), 16611671.

Irving, D., \& Simmonds, I. (2016). A new method for identifying the Pacific-South American pattern and its influence on regional climate variability. Journal of Climate, (2016).

Kalnay, E., Kanamitsu, M., Kistler, R., Collins, W., Deaven, D., Gandin, L., ... \& Zhu, Y. (1996). The NCEP/NCAR 40-year reanalysis project. Bulletin of the American meteorological Society, 77(3), 437-471.

Karoly, D. J. (1989). Southern hemisphere circulation features associated with El Niño-Southern Oscillation events. Journal of Climate, 2(11), 1239-1252.

Kidson, J. W. (1999). Principal modes of Southern Hemisphere low-frequency variability obtained from NCEP-NCAR reanalyses. Journal of Climate, 12(9), 28082830.

Kobayashi, S., Ota, Y., Harada, Y., Ebita, A., Moriya, M., Onoda, H., ... \&Miyaoka, K. (2015). The JRA-55 reanalysis: General specifications and basic characteristics. 気象集誌. 第 2 輯, 93(1)，5-48. 
Krishnamurthy, L., \& Krishnamurthy, V. (2014). Influence of PDO on South Asian summer monsoon and monsoon-ENSO relation. Climate dynamics, 42(9-10), 23972410.

Latif, M., \& Barnett, T. P. (1994). Causes of decadal climate variability over the North Pacific and North America. Science, 266(5185), 634-637.

Latif, M., \& Barnett, T. P. (1996). Decadal climate variability over the North Pacific and North America: Dynamics and predictability. Journal of Climate,9(10), 24072423.

Mantua, N. J., Hare, S. R., Zhang, Y., Wallace, J. M., \& Francis, R. C. (1997).A Pacific interdecadal climate oscillation with impacts on salmon production. Bulletin of the american Meteorological Society, 78(6), 1069-1079.

Mantua, N. J., \& Hare, S. R. (2002). The Pacific decadal oscillation. Journal of oceanography, 58(1), 35-44.

Marengo, J. A. (2004). Interdecadal variability and trends of rainfall across the Amazon basin. Theoretical and applied climatology, 78(1-3), 79-96.

Mo, K. C. (2000). Relationships between low-frequency variability in the Southern Hemisphere and sea surface temperature anomalies. Journal of Climate, 13(20), 3599-3610.

Mo, K. C., \& White, G. H. (1985).Teleconnections in the southern hemisphere. Monthly Weather Review, 113(1), 22-37.

Mo, K. C., \& Ghil, M. (1987). Statistics and dynamics of persistent anomalies. Journal of the Atmospheric Sciences, 44(5), 877-902.

Mo, K. C., \& Higgins, R. W. (1998). Tropical convection and precipitation regimes in the western United States. Journal of climate, 11(9), 2404-2423.

Mo, K. C., \& Paegle, J. N. (2001). The Pacific-South American modes and their downstream effects. International Journal of Climatology, 21(10), 1211-1229.

Mochizuki, T., Ishii, M., Kimoto, M., Chikamoto, Y., Watanabe, M., Nozawa, T., ... \& Toyoda, T. (2010). Pacific decadal oscillation hindcasts relevant to near-term climate prediction. Proceedings of the National Academy of Sciences, 107(5), 18331837.

Robertson, A. W., \& Mechoso, C. R. (2000). Interannual and interdecadal variability of the South Atlantic convergence zone. Monthly weather review,128(8), 29472957.

Schneider, Udo; Becker, Andreas; Finger, Peter; Meyer-Christoffer, Anja; Rudolf, Bruno; Ziese, Markus (2011): GPCC Full Data Reanalysis Version 6.0 at $2.5^{\circ}$ : Monthly Land-Surface Precipitation from Rain-Gauges built on GTS-based and Historic Data. DOI: 10.5676/DWD_GPCC/FD_M_V7_250

Silva, C.B (2012) Variabilidade climática nos oceanos e a vazão fluvial no Pantanal Brasileiro. Dissertação de Mestrado. Faculdade de Filosofia, Letras e Ciências Humanas (FFLCH) da Universidade de São Paulo (USP). 214p.

Silva, C. B., Silva, M. E. S., \& Ambrizzi, T. (2016). Climatic variability of river outflow in the Pantanal region and the influence of sea surface temperature. Theoretical and Applied Climatology, 1-13. 
Wallace, J. M., \& Gutzler, D. S. (1981). Teleconnections in the geopotential height field during the Northern Hemisphere winter. Monthly Weather Review, 109(4), 784-812.

Walker, G. T., \& Bliss, E. W. (1932). World weather. V. Mem. Roy. Meteor. Soc., 4, 53-84. 